\title{
Mesh-preservation approach to treatment of mesh infection after large incisional ventral hernia repair-how I do it
}

\author{
Xuefei Yang ${ }^{1}$, Maimaiti Aihemaiti ${ }^{1}$, Hao Zhang ${ }^{1}$, Li Jiang ${ }^{1}$, Guixi Zhang ${ }^{1}$, Mali Qin ${ }^{1}$, Yiheng Pan $^{1}$, \\ Xiumei Wen ${ }^{1}$, Fion Siu Yin Chan ${ }^{1,2}$, Joe King Man Fan ${ }^{1,2}$ \\ ${ }^{1}$ Department of Surgery, The University of Hong Kong-Shenzhen Hospital, Shenzhen 518053, China; ${ }^{2}$ Department of Surgery, The University of \\ Hong Kong, Hong Kong, China \\ Contributions: (I) Conception and design: X Yang; (II) Administrative support: H Zhang, G Zhang, L Jiang, M Qin, Y Pan, X Wen; (III) Provision \\ of study materials or patients: X Yang; (IV) Collection and assembly of data: X Yang; (V) Data analysis and interpretation: X Yang; (VI) Manuscript \\ writing: All authors; (VII) Final approval of manuscript: All authors. \\ Correspondence to: Joe King Man Fan, MBBS, MS (HKU), FRCSEd, FACS. Department of Surgery, The University of Hong Kong-Shenzhen \\ Hospital, No. 1 Haiyuan 1st Road, Futian District, Shenzhen 518053, China. Email: drjoefan@hku.hk.
}

\begin{abstract}
Mesh infection after large incisional ventral hernia repair is a clinical dilemma in abdominal wall hernia surgery. It is believed foreign material should be removed but it causes secondary trauma to the abdominal wall tissue and might be associated with a higher risk of complications. Currently, there is no consensus on mesh-preservation treatment in cases of mesh infection after hernia repair in general. Herein we present the case of a 27 -year-old male who recovered well from mesh infection after large incisional ventral hernia repair by mesh-preservation approach. The path to success is choice of material of prosthetic mesh; surgical approach of hernia repair, sufficient wound irrigation and drainage, and acquiring sterility of the mesh surface by wound care techniques such as local iodophor packing and vacuum sealing drainage. Clinical cohorts are needed to verify the feasibility of mesh-preservation treatment of mesh infection after large incisional hernia repair.
\end{abstract}

Keywords: Large incisional hernia; mesh infection; mesh-preservation

Submitted Jun 23, 2019. Accepted for publication Oct 17, 2019.

doi: $10.21037 /$ atm.2019.10.82

View this article at: http://dx.doi.org/10.21037/atm.2019.10.82

\section{Introduction}

Huge incisional ventral hernia is defined as hernia defect size $\geq 10 \mathrm{~cm}$ (1), and its surgical correction is considered technically challenging and with a high chance of recurrence. Although repair with prosthesis was proven to reduce hernia recurrences, it associates a series of mesh-related complications like seroma, mesh erosion with sinus formation, chronic pain and discomfort, etc. Even in expert centers, postoperative wound related infective complications as high as $40-50 \%(2-4)$. Seroma formation and mesh infection may also occur as long-term morbidities (5). Nowadays, mesh removal is the preferred management strategy for mesh infection after incisional hernia repair (6-8), which inevitably causes secondary trauma to the abdominal wall tissue and increases the risk of recurrence and other morbidities. Repair of rerecurrent hernia subsequent to mesh removal is even more technically demanding and which usually requires prolonged hospitalization and high medical expenses without promising results. We hereby report a case of huge incisional ventral hernia repair followed by delayed massive effusion around the mesh with infection and wound dehiscence, was eventually treated without the need of mesh removal — by a combination of wound irrigation and vacuum sealing suction drainage. At present, there are only a few reports on mesh-preserving treatment of mesh infection after hernia repair (9-11), and more cohort trials are needed to validate the mesh-preserving treatment strategy for mesh infection after ventral hernia repair. 


\section{Patient demographics}

The patient was a 27 -year-old male who suffered from sigmoid colon perforation due to blunt abdominal injury and underwent exploratory laparotomy with sigmoid colostomy performed 3 years ago, which was complicated by surgical site infection. And the patient underwent wound dressing and debridement, subsequent secondary suture of infected wound, finally with closure of sigmoid stoma. After the second operation, a reducible mass was found over the right paramedian abdominal wound and gradually enlarged, which caused distension discomfort but no other symptoms. There was an incisional hernia over the right lower

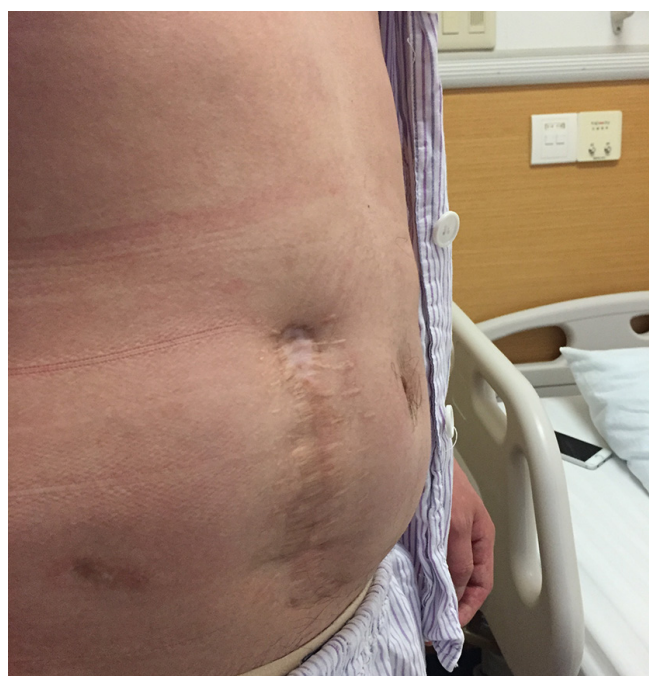

Figure 1 Preoperative appearance of abdominal wall. quadrant, with defect measuring $10 \mathrm{~cm} \times 12 \mathrm{~cm}$ clinically, and the hernia was completely reduced at supine position. The patient had no other significant past medical history.

\section{Pre-operative preparation}

Abdominal contrast CT scan showed an abdominal wall fascial defect in the right lower quadrant, measuring $8.5 \mathrm{~cm} \times$ $11.3 \mathrm{~cm}$, and the small bowel was bulged into the subcutaneous layer to form a large incisional hernia (Figures 1,2). A $15 \mathrm{~cm} \times$ $30 \mathrm{~cm}$ partial absorbable composite mesh was used to ensure an overlapping of 3-5 cm around the original defect by open approach.

\section{Equipment and consumables}

Routine instruments for laparotomy were used. Two/ zero polypropylene non-absorbable suture (Prolene*, Ethicon*), 4/0 monofilament absorbable suture $\left(\right.$ Maxon $^{\mathrm{TM}}$, Covidien $\left.^{\mathrm{TM}}\right), 15 \mathrm{~cm} \times 30 \mathrm{~cm}$ partial absorbable composite mesh (Ultrapro ${ }^{\mathrm{TM}}$, Monocryl ${ }^{\mathrm{TM}}$, Prolene ${ }^{\mathrm{TM}}$, Ethicon $^{\circledR}$ ), the external drainage system $\left(\right.$ Pupunch $^{\circledR}$, Branden $^{\circledR}$ ) were used.

\section{Procedure}

The patient was placed in supine position after general anesthesia, chlorohexidine and alcohol solution were used for abdominal wall disinfection. A right lower abdominal incision, approximately $13 \mathrm{~cm}$ long, was made along the original incision scar. The hernia sac was carefully separated from the subcutaneous tissue to be kept intact, and the
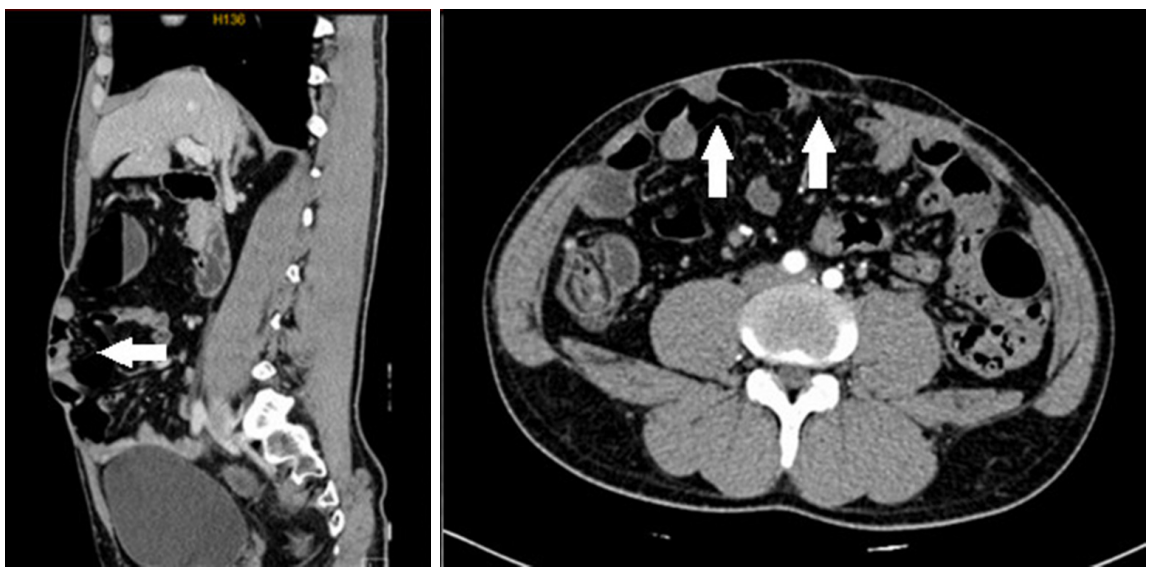

Figure 2 Contrast CT scan showed a large incisional hernia in the right lower quadrant, with the maximum distance between the sagittal and transverse planes measuring $11.3 \mathrm{~cm} \times 8.5 \mathrm{~cm}$. 


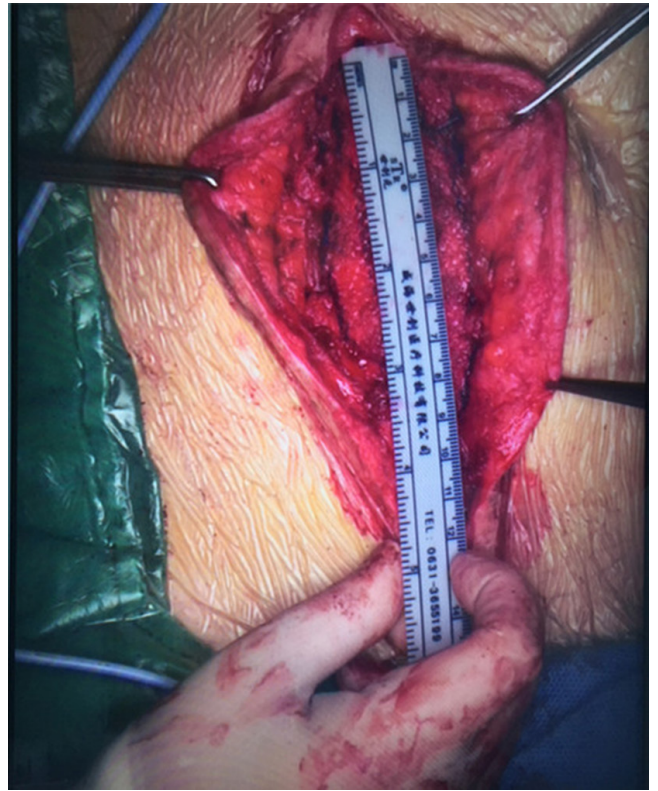

Figure 3 After the mesh and the defect margin were joint with a suture, size of the abdominal wall defect on the mesh surface was reduced to $9 \mathrm{~cm} \times 4 \mathrm{~cm}$.

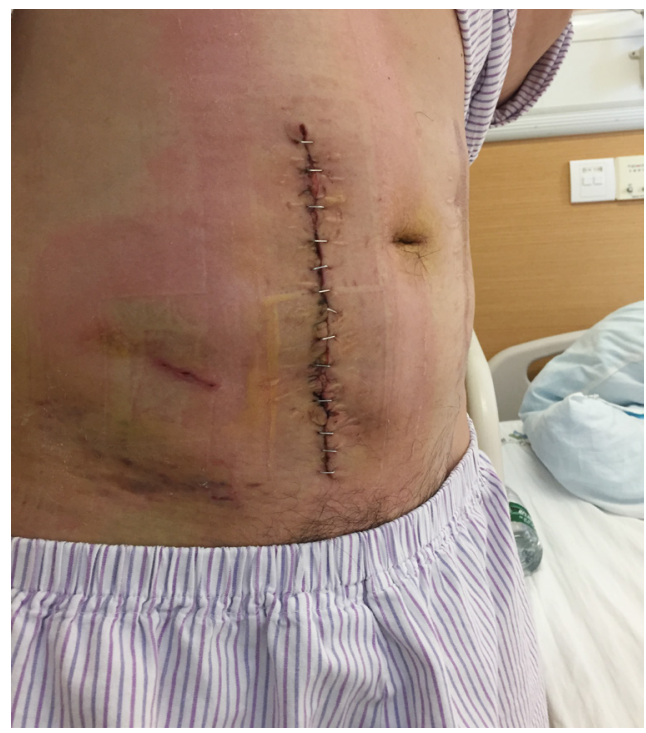

Figure 4 Appearance of the incision and the abdominal wall on the $5^{\text {th }}$ day after surgery.

perforated part, if any, was immediately sutured with $4 / 0$ monofilament absorbable suture to maintain the integrity of the hernia sac. The hernia sac was completely separated from the skin to the edge of the hernia defect, and it measured $12 \mathrm{~cm} \times 8 \mathrm{~cm}$ in diameter. Extra-peritoneal hernia

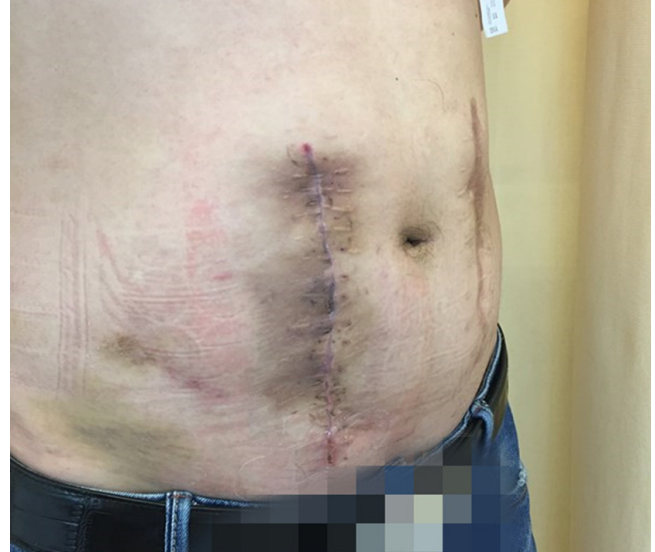

Figure 5 Color of the skin at the incision became deeper on the $17^{\text {th }}$ day after surgery.

sac dissection continued, under the abdominal muscle layer, about $3 \mathrm{~cm}$ to the edge of the fascial defect. The mesh was trimmed into $14 \mathrm{~cm} \times 18 \mathrm{~cm}$ oval shape and was positioned on the surface of the hernia sac, with its edge inserted into the sub-fascial space with enough coverage to the margin of the defect. The mesh was flattened, and the defect was closed by suture before mesh fixation using $2 / 0$ polypropylene nonabsorbable suture. The abdominal wall defect was reduced to $9 \mathrm{~cm} \times 4 \mathrm{~cm}$ (Figure 3). A disposable external drainage system was placed on the mesh surface, skin was trimmed before it was closed by suture. Operative time was 240 minutes and the intraoperative blood loss was $20 \mathrm{~mL}$.

\section{Postoperative progress}

The postoperative drainage reduced from 225 to $9 \mathrm{~mL} /$ day within 4 days and the drainage tube was removed (Figure 4). The patient had no complaint, the incision healed well, and he was discharged after the stitches were removed on postoperative day 8 .

The patient was followed up in outpatient clinic on postoperative day 17 , the incision healed well, the color of skin around the incision became hyper-pigmented (Figure 5), and the patient had no obvious discomfort. On postoperative day 23 , the patient complained about pain over the operative site, and exudate from the incision, which showed no improvement after dressing change treatment. With the amount of exudate increased, a subcutaneous cavity was formed (Figure 6). Although the dressing was changed daily with iodophor gauze packing, the amount 


\section{Page 4 of 7}

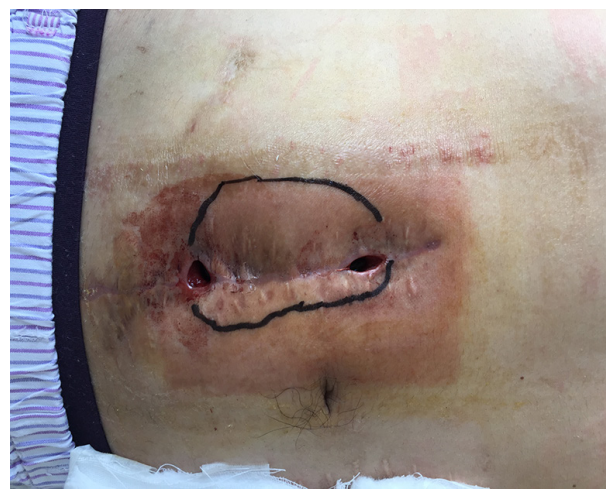

Figure 6 Appearance of the incision on the $35^{\text {th }}$ day after surgery, underline is the range of subcutaneous cavity.

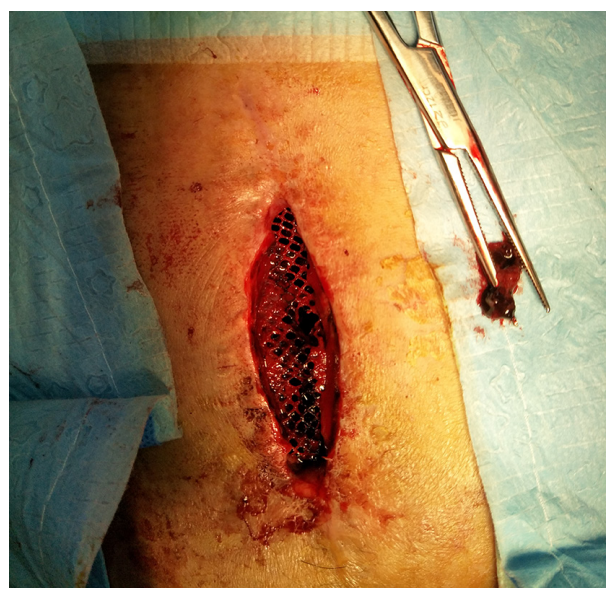

Figure 7 Effusion, clots and necrotic tissue were observed during debridement on the $40^{\text {th }}$ day after surgery.

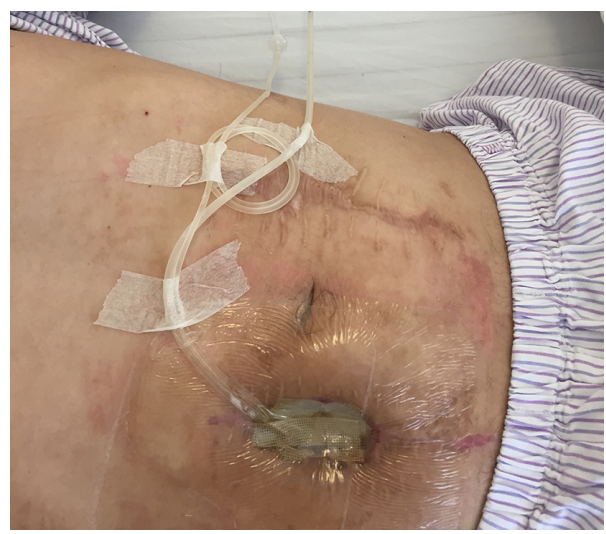

Figure 8 Continuous wound irrigation and vacuum sealing drainage were applied on the $7^{\text {th }}$ day after the second operation.

\section{Yang et al. Mesh-preservation treatment of mesh infection}

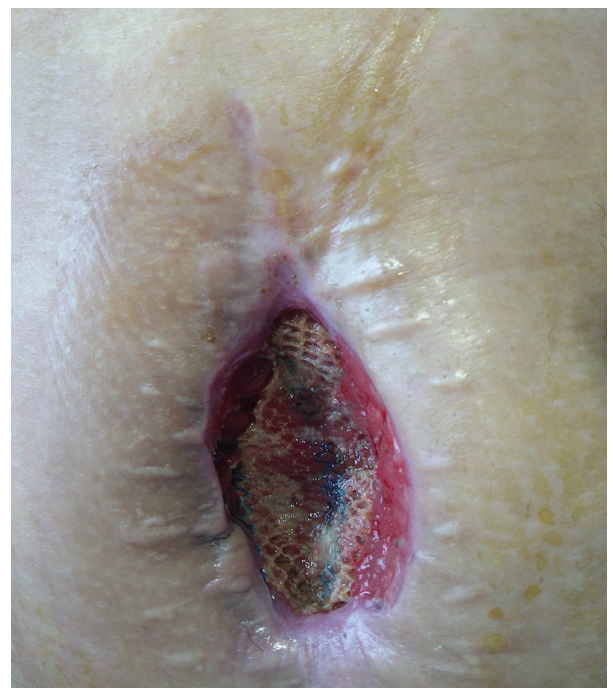

Figure 9 The wound was clean, granulation tissue started growing and the mesh was located on the wound surface.

of exudate still increased, and no bacteria was found in the exudate swab culture. On the $39^{\text {th }}$ day after surgery, ultrasonic examination showed a limited liquid anechoic zone, with clean boundary, measuring $9.4 \mathrm{~cm} \times 4.6 \mathrm{~cm} \times$ $11.4 \mathrm{~cm}$ within the subcutaneous soft tissue in the surgical site.

Surgical debridement and drainage were performed under general anesthesia on postoperative day 40, which found that there was about $80 \mathrm{~mL}$ of dark red jelly like necrotic tissue and old clots accumulated beneath the mesh (Figure 7). The mesh was preserved, the effusion was removed, the mesh space was irrigated with a copious amount of normal saline, and iodophor gauze was packed into the incision after complete hemostasis, then the wound was covered by sterile dressings. Bacterial culture of the effusion found moderate amount of coagulase-negative staphylococci and propionibacterium.

After the second operation, clindamycin was given orally for 1 week, and the dressing was changed with iodophor gauze packing for 4 days. Starting from the $5^{\text {th }}$ day, continuous wound irrigation and vacuum sealing drainage were applied (Figure 8). On the $8^{\text {th }}$ day, granulation tissue adherent with the mesh was observed on the wound surface, size of the incision was gradually reduced, and the mesh could be seen on the wound surface (Figure 9). On postoperative day 11 , no bacterial growth was observed in aerobic and 


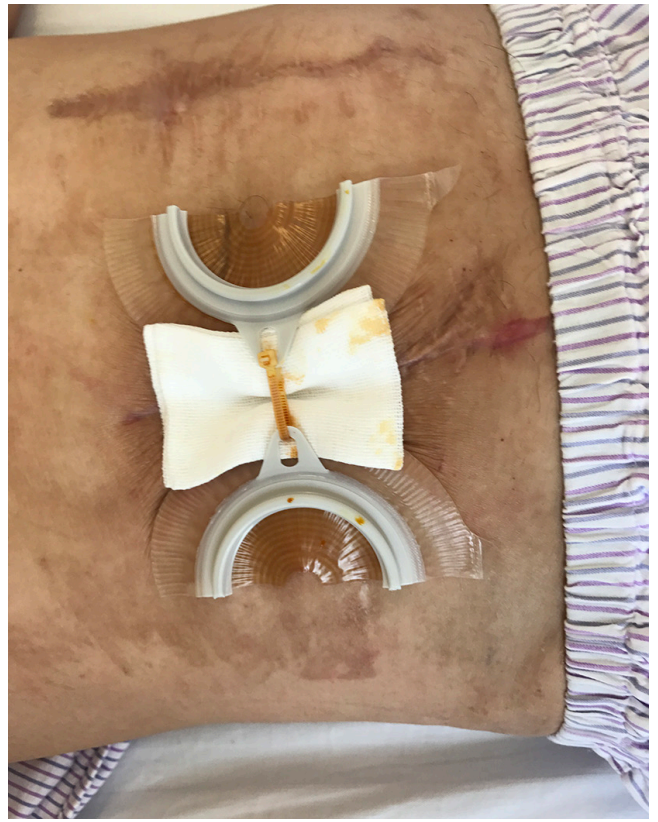

Figure 10 The skin barrier of the ostomy bag and plastic tie band were used to close the incision on the $15^{\text {th }}$ day after the second operation.

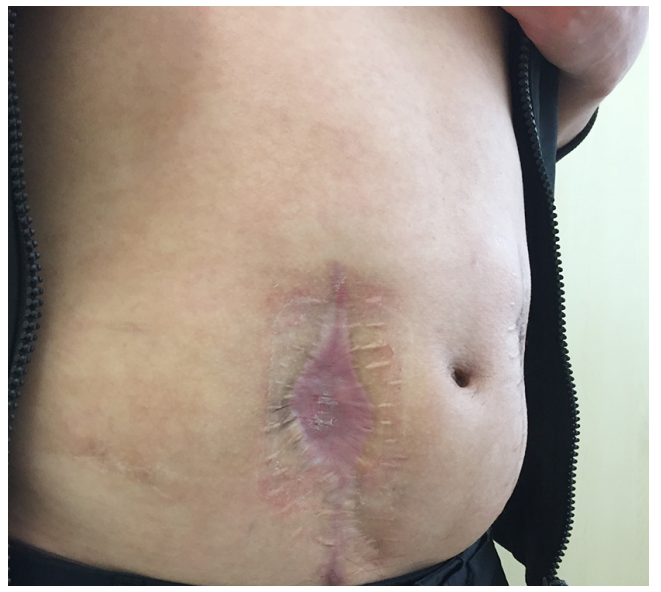

Figure 11 Appearance of the abdominal wall and the incision scar on the $103^{\text {rd }}$ day after the second operation.

anaerobic bacteria culture of the wound extraction. Starting from postoperative day 15 , the skin barrier of the ostomy bag was cut in half and was stick to both sides of the incision, and the skin barriers were pulled together with plastic tie band to close the incision (Figure 10). The wound was cleaned with normal saline daily and was covered with sterile dressing. Size of the incision gradually reduced, the mesh was covered by skin and the incision completely healed by postoperative day 65 (Figure 11). In 22 months of follow-up after hernia repair, the wound completely headed with no pain and no recurrence of incisional hernia.

\section{Tips, tricks and pitfalls}

(I) A drainage tube should be placed on the mesh surface after large incisional hernia repair. After drainage tube removal, however, exudate can continue and accumulate around the mesh and infection may occur: which might be related to the size of mesh, extend of dissection and the ischemic necrosis of the skin over the operative site due to too thin skin flap.

(II) The patient in this case was thin, with a body mass index (BMI) of 21.8 and less subcutaneous fat before surgery. When the hernia sac was separated from the subcutaneous tissue, very thin skin flap was left covering the surgical site, and the thinnest part of the skin on both sides of the incision was trimmed before suture. On postoperative day 17 , however, the skin at the incision margin gradually darkened, followed by necrosis. Therefore, in patients with thin subcutaneous fat, due attention should be paid to the changes in the skin of surgical site even if the incision healed well in the early postoperative period.

(III) Selection of mesh and the method of mesh positioning were also related to the subsequent efficacy of the treatment. We chose macroporous mesh and placed it extraperitoneally (sublay), with the peritoneum remained intact. When the mesh was infected, the effusion was confined to the extraperitoneal layer and did not affect the abdominal viscera. Macroporous mesh had certain advantage in thorough debridement and drainage of the mesh-positioned area without mesh removal, avoiding accumulation of effusion and necrotic tissue, which was advantageous for retaining sterility of the mesh space by dressing change treatment. Abdominal viscera were protected by intact peritoneal isolation, allowing the application of subsequent debridement and vacuum sealing drainage of the wound. Therefore, it is necessary to give serious consideration to the possibility of mesh infection before performing large incisional hernia repair, and proper precautions should be taken when deciding the type of mesh as well as mesh positioning.

(IV) Thorough debridement and drainage should be carried out as soon as possible when extensive effusion on mesh surface when high clinical suspicion of mesh 
infection. During the subsequent treatment, efforts should be made to maintain the sterility of the mesh, such as iodophor gauze packing for 3-4 days and oral use of antibiotics as appropriate. We believe that early iodophor gauze packing may play an important role in obtaining operative site sterility. After wound contraction and the growth of granulation tissues, vacuum sealing drainage can be used. In the meantime, continuous irrigation with sterile saline will keep the wound surface moist, clean and free of effusion to facilitate wound healing.

(V) During the wound healing process, the mesh was held up by granulation tissue and was continuously located on the wound surface. Strict aseptic technique was required during the dressing change process to maintain the sterility of the wound and the mesh, and finally the granulation tissue and the skin embedded the mesh to complete wound healing.

(VI) In mesh infection after large incisional hernia repair, it is still possible to obtain wound healing without mesh removal by sufficient irrigation and drainage as well as subsequent wound treatment. Meshpreserving treatment can avoid secondary trauma to the abdominal wall tissue, reducing the risk of hernia recurrence and other complications such as abdominal viscera injury, and it has special significance for large incisional hernia with high surgical difficulty. However, more clinical trials are needed for its mechanism and treatment process.

\section{Discussion}

In large incisional ventral hernia repair, extensive tissue separation is needed for large mesh placement, causing increased amount of postoperative tissue exudation and higher risk of mesh infection. Treatment of large mesh infection is very difficult since mesh removal might cause further damage to the abdominal wall, and may also damage abdominal viscera, which could result in new complications, making the subsequent treatment process more difficult, painful and expensive. In the case of mesh infection after large incisional hernia repair, the above risks and injuries can be avoided if wound healing is obtained without mesh removal, which is of great significance to the patient. The efficacy of mesh-preserving treatment is influenced by multiple factors during the process, such as mesh selection, placement method, precautionary measures and follow-up observations, which must be fully prepared before surgery.
When mesh infection is detected, thorough debridement should be carried out as early as possible and endeavour to obtain the sterility of the mesh surface, and vacuum sealing drainage should be applied to assist the mesh-preserved healing process, protecting the patient from further damage. There are few comprehensive studies on mesh-preserving treatment of mesh infection after large incisional hernia repair, which is completely feasible from our experience, and more clinical trials with larger sample size are needed for further verification of our conclusion. In the meantime, there is a vast space for innovation in specific treatment methods such as infection control, wound cleaning and promoting tissue healing.

\section{Acknowledgments}

None.

\section{Footnote}

Conflicts of Interest: The authors have no conflicts of interest to declare.

Ethical Statement: The authors are accountable for all aspects of the work in ensuring that questions related to the accuracy or integrity of any part of the work are appropriately investigated and resolved. Institutional Review Board in our center waived the approval process and the informed consent of case photos for single patient if it contains surgical procedures or techniques with no individual identifier and intended risk for individual patient.

\section{References}

1. Muysoms FE, Miserez M, Berrevoet F, et al. Classification of primary and incisional abdominal wall hernias. Hernia 2009; 13:407-14.

2. Bikhchandani J, Fitzgibbons RJ Jr. Repair of giant ventral hernias. Adv Surg 2013;47:1-27.

3. Mathes T, Walgenbach M, Siegel R. Suture versus mesh repair in primary and incisional ventral hernias: a systematic review and meta-analysis. World J Surg 2016;40:826-35.

4. Helgstrand F, Rosenberg J, Kehlet H, et al. Nationwide prospective study of outcomes after elective incisional hernia repair. J Am Coll Surg 2013;216:217-28.

5. Kokotovic D, Bisgaard T, Helgstrand F. Long-term recurrence and complications associated with elective 
incisional hernia repair. JAMA 2016;316:1575-82.

6. Bueno-Lledó J, Torregrosa-Gallud A, Carreño-Saénz $\mathrm{O}$, et al. Partial versus complete removal of the infected mesh after abdominal wall hernia repair. Am J Surg 2017;214:47-52.

7. Hanna M, Dissanaike S. Mesh ingrowth with concomitant bacterial infection resulting in inability to explant: a failure of mesh salvage. Hernia 2015;19:339-44.

8. Liu FD, Li JY, Yao S, et al. A retrospective analysis of surgical treatment of mesh infection after repair of ventral hernia or defect. Genet Mol Res 2015;14:14387-95.

Cite this article as: Yang $X$, Aihemaiti $M$, Zhang $H$, Jiang L, Zhang G, Qin M, Pan Y, Wen X, Chan FSY, Fan JKM. Meshpreservation approach to treatment of mesh infection after large incisional ventral hernia repair-how I do it. Ann Transl Med 2019;7(22):698. doi: 10.21037/atm.2019.10.82
9. Meagher H, Clarke Moloney M, Grace PA. Conservative management of mesh-site infection in hernia repair surgery: a case series. Hernia 2015;19:231-7.

10. Kao AM, Arnold MR, Augenstein VA, et al. Prevention and treatment strategies for mesh infection in abdominal wall reconstruction. Plast Reconstr Surg 2018;142:149S-55S.

11. Nobaek S, Rogmark P, Petersson U. Negative pressure wound therapy for treatment of mesh infection after abdominal surgery: long-term results and patient-reported outcome. Scand J Surg 2017;106:285-93. 